\title{
Isomonodromy for the Degenerate Fifth Painlevé Equation
}

\author{
Primitivo B. ACOSTA-HUMÁNEZ ${ }^{\dagger}$, Marius VAN DER PUT ${ }^{\ddagger}$ and Jaap TOP $\ddagger$ \\ † Universidad Simón Bolívar, Barranquilla, Colombia \\ E-mail: primitivo.acosta@unisimonbolivar.edu.co \\ $\ddagger$ University of Groningen, Groningen, The Netherlands \\ E-mail: m.van.der.put@rug.nl, j.top@rug.nl
}

Received December 12, 2016, in final form May 01, 2017; Published online May 09, 2017

https://doi.org/10.3842/SIGMA.2017.029

\begin{abstract}
This is a sequel to papers by the last two authors making the Riemann-Hilbert correspondence and isomonodromy explicit. For the degenerate fifth Painlevé equation, the moduli spaces for connections and for monodromy are explicitly computed. It is proven that the extended Riemann-Hilbert morphism is an isomorphism. As a consequence these equations have the Painlevé property and the Okamoto-Painlevé space is identified with a moduli space of connections. Using MAPLE computations, one obtains formulas for the degenerate fifth Painlevé equation, for the Bäcklund transformations.
\end{abstract}

Key words: moduli space for linear connections; irregular singularities; Stokes matrices; monodromy spaces; isomonodromic deformations; Painlevé equations

2010 Mathematics Subject Classification: 33E17; 14D20; 14D22; 34M55

\section{Introduction}

In the series of papers $[17,18,19,21,22]$ on isomonodromy families for Painlevé equations the cases $\mathrm{P}_{\mathrm{I}}-\mathrm{P}_{\mathrm{IV}}$ are treated. Here we apply our methods to $\operatorname{deg} \mathrm{P}_{\mathrm{V}}$, the degenerate fifth Painlevé equation. We hope to extend this in a later paper to $\mathrm{P}_{\mathrm{V}}$. We now describe the method of the Riemann-Hilbert correspondence for $\operatorname{deg}_{\mathrm{V}}$, following closely [21, 22].

The degenerate fifth Painlevé equation $\operatorname{deg} \mathrm{P}_{\mathrm{V}}\left(\theta_{0}, \theta_{1}\right)$ depends on two parameters $\theta_{0}, \theta_{1}$ and the corresponding isomonodromy family is given, to begin with, by the $\operatorname{set} \mathbf{S}\left(\theta_{0}, \theta_{1}\right)$ of differential modules $M$ over $\mathbb{C}(z)$ defined by: $\operatorname{dim} M=2$; the exterior product $\Lambda^{2} M$ is trivial; the points 0,1 are regular singular with local exponents $\pm \frac{\theta_{0}}{2}$ and $\pm \frac{\theta_{1}}{2}$. Finally $z=\infty$ is irregular singular with Katz invariant $\frac{1}{2}$, which means that the 'generalized eigenvalues' at $z=\infty$ are $\pm t \cdot z^{1 / 2}$ with $t \in \mathbb{C}^{*}$.

This set is made into an algebraic variety $\mathcal{M}\left(\theta_{0}, \theta_{1}\right)$ which is a moduli space for connections on a fixed bundle on $\mathbb{P}^{1}$ of rank two and degree -1 with prescribed data (see Section 2.1) at $z=0,1, \infty$. If $\theta_{0} \neq 0, \theta_{1} \neq 0$, then $\mathcal{M}\left(\theta_{0}, \theta_{1}\right)$ is a fine modul space and is smooth. For $\theta_{0}=0$ and/or $\theta_{1}=0$, the moduli problem will be changed by adding "an invariant line". This is called a parabolic structure in the literature, see $[4,5,6,7]$. It leads to a fine moduli space $\mathcal{M}^{+}\left(\theta_{0}, \theta_{1}\right)$ which is a desingularisation of $\mathcal{M}\left(\theta_{0}, \theta_{1}\right)$.

The analytic data attached to modules in $\mathbf{S}\left(\theta_{0}, \theta_{1}\right)$ are two monodromy matrices and one Stokes matrix. They produce a 'monodromy space' $\mathcal{R}\left(s_{0}, s_{1}\right)$ depending on $s_{0}=e^{\pi i \theta_{0}}+e^{-\pi i \theta_{0}}$ and $s_{1}=e^{\pi i \theta_{1}}+e^{-\pi i \theta_{1}}$. For $s_{0} \neq \pm 2$ and $s_{1} \neq \pm 2$ the monodromy space is a fine moduli space and is smooth. It is in fact a smooth affine cubic surface with three lines at infinity. For the other cases one changes the moduli problem by adding an 'invariant line'. The fine moduli space $\mathcal{R}^{+}\left(s_{0}, s_{1}\right)$ for these new data is a minimal resolution of $\mathcal{R}\left(s_{0}, s_{1}\right)$. 
The Stokes matrix attached to a module in $\mathbf{S}\left(\theta_{0}, \theta_{1}\right)$ depends on the choice of a summation direction at $z=\infty$. This direction has to be different from the singular direction at $z=\infty$ which turns around $\infty$ for $t$ varying in $T:=\mathbb{C}^{*}$. Therefore there is a locally, with respect to $t \in \mathbb{C}^{*}$, defined analytic Riemann-Hilbert map RH: $\mathcal{M}^{+}\left(\theta_{0}, \theta_{1}\right) \rightarrow \mathcal{R}^{+}\left(s_{0}, s_{1}\right)$ with $s_{0}=e^{\pi i \theta_{0}}+e^{-\pi i \theta_{0}}$ and $s_{1}=e^{\pi i \theta_{1}}+e^{-\pi i \theta_{1}}$. After replacing $T$ by its universal covering $\tilde{T}=\mathbb{C}$ the morphism $\mathrm{RH}$ is well defined. The main result is that the extended Riemann-Hilbert map

$$
\mathrm{RH}^{+}: \mathcal{M}^{+}\left(\theta_{0}, \theta_{1}\right) \times_{T} \tilde{T} \rightarrow \mathcal{R}^{+}\left(s_{0}, s_{1}\right) \times \tilde{T}
$$

is an analytic isomorphism. It follows from this that $\operatorname{deg}_{\mathrm{V}}$ has the Painlevé property and that $\mathcal{M}^{+}\left(\theta_{0}, \theta_{1}\right)$ coincides with the Okamoto-Painlevé space (see [23, 24, 25] for this subject). The explicit computations of the spaces $\mathcal{M}^{+}\left(\theta_{0}, \theta_{1}\right)$ and $\mathcal{R}^{+}\left(s_{0}, s_{1}\right)$ lead, using MAPLE, to formulas for $\operatorname{deg} \mathrm{P}_{\mathrm{V}}$ and for the Bäcklund transformations.

We note that our definition of $\operatorname{deg}_{\mathrm{V}}$ is not quite the same as the 'classical' degenerate fifth Painlevé equation. The close relation between the two is given in Section 3. We were informed by Y. Ohyama about the 'equivalence' between $\operatorname{deg} \mathrm{P}_{\mathrm{V}}$ and $\mathrm{P}_{\mathrm{III}}\left(D_{6}\right)$ found by V.I. Gromak [3]. A Hamiltonian for $\mathrm{P}_{\mathrm{III}}\left(D_{6}\right)$ is

$$
\frac{1}{t}\left(q^{2} p^{2}-\left(q^{2}-(\alpha+\beta) q-t\right) p-\alpha q\right)
$$

The degrees in $p$ and $q$ are $\leq 2$. Therefore the Hamilton equations allow to eliminate $p$ (in terms of $q, q^{\prime}$ ) and also to eliminate $q$ (in terms of $\left.p, p^{\prime}\right)$. In the first case one obtains $\mathrm{P}_{\mathrm{III}}\left(D_{6}\right)$ and in the second case the classical degenerate fifth Painlevé equation.

This equivalence does not seem to produce a relation between the isomonodromy families for $\operatorname{deg} \mathrm{P}_{\mathrm{V}}$ and for $\mathrm{P}_{\mathrm{III}}\left(D_{6}\right)$.

Apart from the references given at the beginning of this introduction, several sources discuss geometric aspects of Painlevé equations. Many of these can be found in the references of our papers $[17,18,19,21,22]$. Classical' papers on the subject are $[8,9,12,13,14,15,16]$. Especially relevant for the present text are the paper by Ohyama and Okumura [11], Witte's paper [26]. The book [2] by Fokas, Its, Kapaev, and Novokshenov discusses more analytic aspects of the Riemann-Hilbert correspondence, but it does not discuss the degenerate fifth Painlevé equation. Finally, we mention the recent paper [1] by Chekhov, Mazzocco, and Rubtsov which also provides an interesting geometric appoach and overview.

\section{The moduli space $\mathcal{M}\left(\theta_{0}, \theta_{1}\right)$ of connections}

\subsection{Definition of $\mathrm{S}\left(\theta_{0}, \theta_{1}\right)$}

An element of the set $\mathbf{S}\left(\theta_{0}, \theta_{1}\right)$ is (the isomorphy class of) a tuple $\left(M, \theta_{0}, \theta_{1}, t\right)$ where $M$ is a differential module over $\mathbb{C}(z)$ such that $\operatorname{dim} M=2 ; \operatorname{det} M:=\Lambda^{2} M$ is the trivial module; $M$ has three singular points $0,1, \infty$ and their Katz invariants are $r(0)=0, r(1)=0, r(\infty)=1 / 2$. Further, the singularities are represented:

- at $z=0$ by $\frac{d}{d z}+\frac{1}{z}\left(\begin{array}{cc}\omega_{0} & * \\ 0 & -\omega_{0}\end{array}\right)$ with $\omega_{0}=\frac{\theta_{0}}{2}$ and $\theta_{0}, * \in \mathbb{C}$;

- at $z=1$ by $\frac{d}{d z}+\frac{1}{z-1}\left(\begin{array}{cc}\omega_{1} & * \\ 0 & -\omega_{1}\end{array}\right)$ with $\omega_{1}=\frac{\theta_{1}}{2}$ and $\theta_{1}, * \in \mathbb{C}$;

- at $z=\infty$ by $z \frac{d}{d z}+\left(\begin{array}{cc}\omega_{\infty} & 0 \\ 0 & -\omega_{\infty}\end{array}\right)$ with $\omega_{\infty}=t z^{1 / 2}$ and $t \in \mathbb{C}^{*}$.

Let $\partial$ denote the given differential operator on $M$, corresponding with the derivation $\frac{d}{d z}$ on $\mathbb{C}(z)$, i.e., $\partial(f m)=\frac{d f}{d z} m+f \partial(m)$ for $f \in \mathbb{C}(z)$ and $m \in M$. 
The condition at $z=0$ means that $\mathbb{C}((z)) \otimes M$ has a $\mathbb{C}[[z]]$-lattice with a basis such that the matrix of $z \partial$ is $\left(\begin{array}{cc}\omega_{0} & * \\ 0 & -\omega_{0}\end{array}\right)$. For $\theta_{0} \neq 0$ one can always take $*=0$. For $\theta_{0}=0$, any $*$ is admissible. At $z=1$ the condition is similar for the matrix of $(z-1) \partial$.

The condition at $z=\infty$ means that $\mathbb{C}\left(\left(z^{-1 / 2}\right)\right) \otimes M$ has a basis for which $z \partial$ has the matrix $\left(\begin{array}{cc}\omega_{\infty} & 0 \\ 0 & -\omega_{\infty}\end{array}\right)$. In the construction of the moduli space $\mathcal{M}\left(\theta_{0}, \theta_{1}\right)$ this matrix will be changed in a matrix defined over $\mathbb{C}\left(\left(z^{-1}\right)\right)$.

There is an obvious bijection $\mathbf{S}\left(\theta_{0}, \theta_{1}\right) \rightarrow \mathbf{S}\left(\theta_{0}+a, \theta_{1}+b\right)$ for any $a, b \in 2 \mathbb{Z}$, obtained by changing the lattices for $z=0$ and $z=1$.

\subsection{Choosing connections}

We represent $M \in \mathbf{S}\left(\theta_{0}, \theta_{1}\right)$ by a connection $\nabla: \mathcal{V} \rightarrow \Omega([0]+[1]+2[\infty]) \otimes \mathcal{V}$, where $\mathcal{V}$ is a vector bundle on $\mathbb{P}^{1}$ of rank two. Thus the generic fiber of $\nabla$ is the map $M \rightarrow M \otimes_{\mathbb{C}(z)} \mathbb{C}(z) d z$, given by $m \mapsto \partial(m) d z$.

The vector bundle $\mathcal{V}$ is determined by the choice of the lattices at every point of $\mathbb{P}^{1}$. For the points $z=a \neq 0,1, \infty$ the lattice in $\mathbb{C}((z-a)) \otimes M$ is the $\mathbb{C}[[z-a]]$-module generated by $\operatorname{ker}(\partial, \mathbb{C}((z-a)) \otimes M)$. For the points $z=0$ and $z=1$ we choose the lattices corresponding to the given $\theta_{0}, \theta_{1}$. In other words, the characteristic polynomials of the matrices of $z \partial$ and $(z-1) \partial$ are prescribed by $X^{2}-\frac{\theta_{0}^{2}}{4}$ and $X^{2}-\frac{\theta_{1}^{2}}{4}$.

At $z=\infty$ the situation is more complicated. An invariant lattice at $\infty$ is represented over the differential field $\mathbb{C}\left(\left(z^{-1 / 2}\right)\right)$ by $z \frac{d}{d z}+\left(\begin{array}{cc}t z^{1 / 2} & 0 \\ 0 & -t z^{1 / 2}\end{array}\right)$. We need an expression over the field $\mathbb{C}\left(\left(z^{-1}\right)\right)$ or "invariant lattices" for $\mathbb{C}\left(\left(z^{-1}\right)\right) \otimes M$. For Katz invariant $1 / 2$ or 1 at $\infty$, a lattice $\Lambda$ is called invariant if $z \partial \Lambda \subset \Lambda$.

We adopt here the terminology [20] for the classification of differential modules over $\mathbb{C}\left(\left(z^{-1}\right)\right)$.

The formal solution space $V$ at $z=\infty$ is described as $V=V_{q} \oplus V_{-q}=\mathbb{C} e_{1} \oplus \mathbb{C} e_{2}$ with $q=t z^{1 / 2}$ and the formal monodromy $\gamma$ is given by $\gamma\left(e_{1}\right)=e_{2}$ and $\gamma\left(e_{2}\right)=-e_{1}$. Indeed, the determinant of $M$ is trivial and hence $\operatorname{det}(\gamma)=1$. The (formal local) differential module $\mathbb{C}\left(\left(z^{-1}\right)\right) \otimes M$ and its invariant lattices are now obtained by considering the invariants of $\mathcal{U} \otimes_{\mathbb{C}} V$ (here $\mathcal{U}$ is the universal Picard-Vessiot ring for $\left.\mathbb{C}\left(\left(z^{-1}\right)\right)\right)$ under the actions of the differential automorphisms of $\mathcal{U}$ over $\mathbb{C}\left(\left(z^{-1}\right)\right)$. A computation yields invariant lattices $\Lambda_{1}$ and $\Lambda_{2}$ represented by

$$
z \frac{d}{d z}+\left(\begin{array}{cc}
-\frac{1}{4} & t z \\
t & \frac{1}{4}
\end{array}\right) \quad \text { and } \quad z \frac{d}{d z}+\left(\begin{array}{cc}
-\frac{3}{4} & t z \\
t & -\frac{1}{4}
\end{array}\right)
$$

All lattices are given by $z^{n} \Lambda_{1}$ and $z^{n} \Lambda_{2}$ with $n \in \mathbb{Z}$.

Remark 2.1. By conjugation with the constant matrix $\left(\begin{array}{ll}t & 0 \\ 0 & 1\end{array}\right)$ one can change these formulas into

$$
z \frac{d}{d z}+\left(\begin{array}{cc}
-\frac{1}{4} & t^{2} z \\
1 & \frac{1}{4}
\end{array}\right) \quad \text { and } \quad z \frac{d}{d z}+\left(\begin{array}{cc}
-\frac{3}{4} & t^{2} z \\
1 & -\frac{1}{4}
\end{array}\right)
$$

We want that $\operatorname{det} \mathcal{V}:=\Lambda^{2} \mathcal{V}$ has degree -1 . The reason is that, in general $\mathcal{V}=O\left(d_{1}\right) \oplus O\left(d_{2}\right)$ with $d_{1} \leq d_{2}$. The differential module $\mathbb{C}\left(\left(z^{-1}\right)\right) \otimes M$ is irreducible because of the ramification at $z=\infty$. Hence $M$ and also the connection on $\mathcal{V}$ is irreducible. This implies (in this special case) that $d_{2}-d_{1} \leq 2$. If we make the natural assumption that $\operatorname{deg} \mathcal{V}=0$, then there are two possibilities for $\mathcal{V}$, namely $O \oplus O$ and $O(-1) \oplus O(+1)$. The construction of a family of connection would involve a construction of a family of vector bundles of rank two and degree 0 on $\mathbb{P}^{1}$. However, we will avoid this by imposing $\operatorname{deg} \mathcal{V}=-1$. Then there is only one possibility for $\mathcal{V}$, namely $O \oplus O(-1)$.

Finally, the degree of $\mathcal{V}$ is -1 precisely for the choice of the lattice $\Lambda_{2}$ corresponding to $z \frac{d}{d z}+\left(\begin{array}{cc}-\frac{3}{4} & t z \\ t & -\frac{1}{4}\end{array}\right)$. 


\subsection{Computation of the connection}

We identify $\mathcal{V}$ with $O e_{1} \oplus O(-[\infty]) e_{2}$ (a subvector bundle of the free (i.e., trivial) vector bundle $\left.O e_{1} \oplus O e_{2}\right)$. Let $D=\nabla_{\frac{d}{d z}}$ and $\tilde{D}:=z(z-1) D$. Then $\tilde{D} e_{1} \in\left\langle 1, z, z^{2}\right\rangle e_{1}+\langle 1, z\rangle e_{2} ; \tilde{D} e_{2} \in$ $\left\langle 1, z, z^{2}, z^{3}\right\rangle e_{1}+\left\langle 1, z, z^{2}\right\rangle e_{2}$. Here $\langle *, *, *\rangle$ denotes the $\mathbb{C}$-vector spaces generated by these $*$ 's. The vector bundle $\mathcal{V}$ has an automorphism group $G$ and the moduli space $\mathcal{M}\left(\theta_{0}, \theta_{1}\right)$ (note that we fix the parameters $\theta_{0}$ and $\theta_{1}$ in this construction), that we are constructing, is obtained by dividing the space of all matrices for the connections by the group $G$. This group consists of the elements $e_{1} \mapsto \lambda e_{1}, e_{2} \mapsto \mu e_{2}+\left(x_{0}+x_{1} z\right) e_{1}$ (with $\lambda, \mu \in \mathbb{C}^{*}, x_{0}, x_{1} \in \mathbb{C}$ ). Further the multiples of the identity act trivially and we have only to consider the automorphisms $e_{1} \mapsto \lambda e_{1}$, $e_{2} \mapsto e_{2}+\left(x_{0}+x_{1} z\right) e_{1}$.

Since the connection is irreducible we have that $\tilde{D} e_{1}=\left\langle 1, z, z^{2}\right\rangle e_{1}+\left(b_{1} z+b_{0}\right) e_{2}$ with $\left(b_{1} z+\right.$ $\left.b_{0}\right) \neq 0$. We consider now two affine parts: $b_{1} \neq 0$ and $b_{0} \neq 0$. The two affine parts are divided out by the action of $G$. These quotients are geometric quotients and they are obtained by normalization of some of the entries of the matrices. One obtains two affine varieties $\mathcal{M}_{1}\left(\theta_{0}, \theta_{1}\right)$ and $\mathcal{M}_{2}\left(\theta_{0}, \theta_{1}\right)$ which are glued to the moduli space $\mathcal{M}\left(\theta_{0}, \theta_{1}\right)$.

\subsubsection{The first affine part $\mathcal{M}_{1}\left(\theta_{0}, \theta_{1}\right)$}

This is obtained by dividing the open subspace $b_{1} \neq 0$ by the action of the group $G$. First one normalizes $b_{1}$ by the automorphism $e_{1} \mapsto \lambda e_{1}$ to $b_{1}=1$. Using $e_{2} \mapsto e_{2}+\left(a_{0}+a_{1} z\right) e_{1}$ one normalizes further to $\tilde{D} e_{1}=a_{0} e_{1}+\left(z+b_{0}\right) e_{2}$ and $\tilde{D} e_{2}=\left(c_{0}+c_{1} z+c_{2} z^{2}+c_{3} z^{3}\right) e_{1}+\left(d_{0}+d_{1} z+\right.$ $\left.d_{2} z^{2}\right) e_{2}$. Now we have to compute the equations between the variables due to the prescription of the three invariant lattices.

For $z=0$. Now $\frac{1}{z-1} \tilde{D}$ has modulo $z=0$ the eigenvalues $\pm \frac{\theta_{0}}{2}$. This yields $a_{0}+d_{0}=0$ and $a_{0} d_{0}-b_{0} c_{0}=-\frac{\theta_{0}^{2}}{4}$.

For $z=1$. Now $\frac{1}{z} \tilde{D}$ has modulo $z=1$ the eigenvalues $\pm \frac{\theta_{1}}{2}$. This yields $a_{0}+d_{0}+d_{1}+d_{2}=0$ and $a_{0}\left(d_{0}+d_{1}+d_{2}\right)-\left(1+b_{0}\right)\left(c_{0}+c_{1}+c_{2}+c_{3}\right)=-\frac{\theta_{1}^{2}}{4}$.

For $z=\infty$. The local basis of $\mathcal{V}$ at $z=\infty$ is $e_{1}, z^{-1} e_{2}$. The operator $\frac{1}{z-1} \tilde{D}$ w.r.t. this local basis is $z \frac{d}{d z}+\left(\begin{array}{cc}\frac{a_{0}}{z-1} & \frac{c_{0}+c_{1} z+c_{2} z^{2}+c_{3} z^{3}}{z(z-1)} \\ z \frac{z+b_{0}}{z-1} & -1+\frac{d_{0}+d_{1} z+d_{2} z^{2}}{z-1}\end{array}\right)$. This should be equivalent to $z \frac{d}{d z}+\left(\begin{array}{cc}\frac{-3}{4} & t z \\ t & \frac{-1}{4}\end{array}\right)$. The characteristic polynomials of the two matrices should be equal modulo $\mathbb{C}\left[\left[z^{-1}\right]\right]$.

Remark 2.2. A more precise computation is needed to verify the correctness of the last statement. On another basis of the lattice, the operator $z \frac{d}{d z}+\left(\begin{array}{cc}\frac{-3}{4} & t z \\ t & \frac{-1}{4}\end{array}\right)$ reads

$$
\left(1+A_{1} z^{-1}+\cdots\right)^{-1} A_{0}^{-1}\left(z \frac{d}{d z}+\left(\begin{array}{cc}
\frac{-3}{4} & t z \\
t & \frac{-1}{4}
\end{array}\right)\right) A_{0}\left(1+A_{1} z^{-1}+\cdots\right),
$$

leading to

$$
z \frac{d}{d z}+A_{0}^{-1}\left(\left(\begin{array}{cc}
\frac{-3}{4} & t z \\
t & \frac{-1}{4}
\end{array}\right)+t\left(\begin{array}{cc}
x_{3} & x_{4}-x_{1} \\
0 & -x_{3}
\end{array}\right)\right) A_{0}
$$

where $A_{0} \in \mathrm{GL}_{2}(\mathbb{C}), x_{1}, x_{3}, x_{4} \in \mathbb{C}$. Thus $d_{2}=0, d_{1}=0, c_{3}=0$ and $c_{2}=t^{2}$.

Finally the connection as matrix differential operator w.r.t. $e_{1}, e_{2}$ reads

$$
\frac{d}{d z}+\frac{1}{z(z-1)}\left(\begin{array}{cc}
a_{0} & c_{0}+c_{1} z+t^{2} z^{2} \\
z+b_{0} & -a_{0}
\end{array}\right)
$$


and there are two equations

$$
a_{0}^{2}+b_{0} c_{0}=\frac{\theta_{0}^{2}}{4}, \quad a_{0}^{2}+\left(1+b_{0}\right)\left(c_{0}+c_{1}+t^{2}\right)=\frac{\theta_{1}^{2}}{4} .
$$

The last equation can be changed into

$$
c_{0}=-\frac{\theta_{0}^{2}}{4}+\frac{\theta_{1}^{2}}{4}-c_{1}-t^{2}-b_{0} c_{1}-b_{0} t^{2}
$$

and this is used to eliminate $c_{0}$. This leaves four variables $a_{0}, b_{0}, c_{1}, t$ and one equation

$$
a_{0}^{2}+b_{0}\left(-\frac{\theta_{0}^{2}}{4}+\frac{\theta_{1}^{2}}{4}-c_{1}-t^{2}-b_{0} c_{1}-b_{0} t^{2}\right)-\frac{\theta_{0}^{2}}{4}=0 .
$$

The above describes the space $\mathcal{M}_{1}\left(\theta_{0}, \theta_{1}\right)$. We note that this space depends only on $\theta_{0}^{2}$ and $\theta_{1}^{2}$. The space $\mathcal{M}_{1}\left(\theta_{0}, \theta_{1}\right)$ is smooth if $\theta_{0} \neq 0$ and $\theta_{1} \neq 0$. For $\theta_{0}=0, \theta_{1} \neq 0$, the singular locus is given by $a_{0}=0, b_{0}=0, c_{1}=-t^{2}+\frac{\theta_{1}^{2}}{4}$. For $\theta_{0} \neq 0, \theta_{1}=0$, the singular locus is given by $a_{0}=0$, $b_{0}=-1, c_{1}=-t^{2}+\frac{\theta_{0}^{2}}{4}$. For $\theta_{0}=\theta_{1}=0$, the singular locus is given by $a_{0}=0, b_{0}\left(b_{0}+1\right)=0$, $c_{1}=-t^{2}$.

Moreover $\mathcal{M}_{1}\left(\theta_{0}, \theta_{1}\right)$ seen as a two dimensional space over the field $\mathbb{C}(t)$ has the same singular locus but now seen as a set of at most two points.

Observation 2.3. For every $a \in \mathbb{C}^{*}$ the closed subspace of $\mathcal{M}_{1}\left(\theta_{0}, \theta_{1}\right)$, defined by $t=a$, is simply connected.

Indeed, the equation

$$
a_{0}^{2}+b_{0}\left(-\frac{\theta_{0}^{2}}{4}+\frac{\theta_{1}^{2}}{4}-c_{1}-a^{2}-b_{0} c_{1}-b_{0} a^{2}\right)-\frac{\theta_{0}^{2}}{4}=0
$$

defines this two-dimensional space. It is mapped to $\mathbb{C}^{2}$ by $\left(a_{0}, b_{0}, c_{1}\right) \mapsto\left(a_{0}, b_{0}\right)$. The fibre is either empty, or a point, or $\mathbb{C}$. Hence it suffices to show that the image $B \subset \mathbb{C}^{2}$ is simply connected. Now $B$ is the union of $X:=\mathbb{C} \times(\mathbb{C} \backslash\{0,-1\})$ and the points $\left( \pm \frac{\theta_{0}}{2}, 0\right)$ and $\left( \pm \frac{\theta_{1}}{2},-1\right)$. The canonical map $\pi_{1}(X, *) \rightarrow \pi_{1}(B, *)$ is surjective. Consider one of the two generators, $s \in[0,1] \mapsto\left(\frac{\theta_{0}}{2}, e^{2 \pi i s}\right)$ of $\pi_{1}(X, *)$. In $B$ this closed path is homotopic to the constant closed path by the homotopy $s, \lambda \in[0,1] \mapsto\left(\frac{\theta_{0}}{2}, \lambda e^{2 \pi i s}\right)$. The same observation can be made for the other generator of $\pi_{1}(X, *)$. Hence $B$ is simply connected.

\subsubsection{The second affine part $\mathcal{M}_{2}\left(\theta_{0}, \theta_{1}\right)$}

This space is obtained by dividing the open subset $b_{0} \neq 0$ by the action of $G$. Now $b_{0} \neq 0$ is normalized by the automorphism $e_{1} \mapsto \lambda e_{1}$ to $b_{0}=1$.

Using $e_{2} \mapsto e_{2}+\left(x_{0}+x_{1} z\right) e_{2}$ one normalizes further to $\tilde{D} e_{1}=a_{2} z^{2} e_{1}+\left(b_{1} z+1\right) e_{2}$ and $\tilde{D} e_{2}=\left(c_{0}+c_{1} z+c_{2} z^{2}+c_{3} z^{3}\right) e_{1}+\left(d_{0}+d_{1} z+d_{2} z^{2}\right) e_{2}$. The equations derived from the prescribed invariant lattices are

For $z=0: d_{0}=0$ and $c_{0}=\frac{\theta_{0}^{2}}{4}$.

For $z=1: a_{2}+d_{1}+d_{2}=0$ and $a_{2}\left(d_{1}+d_{2}\right)-\left(1+b_{1}\right)\left(c_{0}+c_{1}+c_{2}+c_{3}\right)=-\frac{\theta_{1}^{2}}{4}$.

For $z=\infty$ : the operator $\frac{1}{z-1} \tilde{D}$ w.r.t. $e_{1}, z^{-1} e_{2}$ is

$$
z \frac{d}{d z}+\left(\begin{array}{cc}
\frac{a_{2} z^{2}}{z-1} & \frac{c_{0}+c_{1} z+c_{2} z^{2}+c_{3} z^{3}}{z(z-1)} \\
\left(1+b_{1} z\right) \frac{z}{z-1} & -1+\frac{d_{0}+d_{1} z+d_{2} z^{2}}{z-1}
\end{array}\right)
$$


and is equivalent to

$$
z \frac{d}{d z}+\left(\begin{array}{cc}
\frac{-3}{4} & t z \\
t & \frac{-1}{4}
\end{array}\right)
$$

This yields the equations $d_{2}=-a_{2}$ (and from before $d_{1}+d_{2}=-a_{2}$ and thus $d_{1}=0$ ). Further $a_{2}^{2}+b_{1} c_{3}=0$ and $c_{3}=t^{2}-b_{1} c_{2}-a_{2}$ is used to eliminate $c_{3}$. One obtains the equation

$$
c_{1}+c_{2}+t^{2}+b_{1} \frac{\theta_{0}^{2}}{4}+b_{1} c_{1}-a_{2}+\frac{\theta_{0}^{2}}{4}-\frac{\theta_{1}^{2}}{4}=0
$$

which eliminates $c_{2}$. The matrix differential operator w.r.t. the basis $e_{1}, e_{2}$ is now

$$
\frac{d}{d z}+\frac{1}{z(z-1)}\left(\begin{array}{cc}
a_{2} z^{2} & \frac{\theta_{0}^{2}}{4}+c_{1} z+c_{2} z^{2}+\left(t^{2}-b_{1} c_{2}-a_{2}\right) z^{3} \\
1+b_{1} z & -a_{2} z^{2}
\end{array}\right)
$$

in the variables $a_{2}, b_{1}, c_{1}, t$ and one equation $a_{2}^{2}+b_{1}\left(-a_{2}-b_{1} c_{2}+t^{2}\right)=0$ with $c_{2}$ eliminated as above. The above describes the space $\mathcal{M}_{2}\left(\theta_{0}, \theta_{1}\right)$.

The singular locus of this three-dimensional variety is given by $\theta_{1}=0, a_{2}=0, b_{1}=-1$,

$c_{1}=t^{2}-\frac{\theta_{0}^{2}}{4}$ (note that $t \neq 0$ ). As a variety over $\mathbb{C}(t)$ the singularity occurs only for $\theta_{1}=0$ and consists of one point.

The two parts glue to the required space $\mathcal{M}\left(\theta_{0}, \theta_{1}\right)$.

Observation 2.4. For any $a \in \mathbb{C}^{*}$ the closed subspace of $\mathcal{M}\left(\theta_{0}, \theta_{1}\right)$, defined by $t=a$, is simply connected.

Indeed, by Observation 2.3, this holds for the first open affine part of this space. For the second open affine part the same reasoning proves the statement. Van Kampen's theorem finishes the proof.

\subsubsection{Resolving the singularities of $\mathcal{M}\left(\theta_{0}, \theta_{1}\right)$}

For $\theta_{0} \neq 0, \theta_{1} \neq 0$, the space $\mathcal{M}\left(\theta_{0}, \theta_{1}\right)$ is the fine moduli space for the connections on the fixed vector bundle $\mathcal{V}$ of rank 2 and degree -1 with the prescribed singularities. The space $\mathcal{M}\left(\theta_{0}, \theta_{1}\right)$ is smooth and simply connected for any nonzero fixed value of $t$. Its set of closed points $\mathcal{M}\left(\theta_{0}, \theta_{1}\right)(\mathbb{C})$ coincides by construction with $\mathbf{S}\left(\theta_{0}, \theta_{1}\right)$.

For $\theta_{0}=0$ and/or for $\theta_{1}=0$ the space $\mathcal{M}\left(\theta_{0}, \theta_{1}\right)$ has singularities and is no longer a fine moduli space. Consider the case $\theta_{0}=0$ and $\theta_{1} \neq 0$.

A tuple $\left(M, \theta_{0}=0, \theta_{1}, t\right)$ is represented at $z=0$ by a lattice $\Lambda \subset \mathbb{C}((z)) \otimes M$ such that $\delta(\Lambda) \subset \Lambda$ and the action of $\delta:=z D$ on $\Lambda / z \Lambda$ has trace zero and determinant zero. There exists a basis of $\Lambda$ over $\mathbb{C}[[z]]$ such that $\delta$ has the matrix $\left(\begin{array}{ll}0 & 0 \\ 0 & 0\end{array}\right)$ or $\left(\begin{array}{ll}0 & 1 \\ 0 & 0\end{array}\right)$. The space $\mathcal{M}\left(\theta_{0}=0, \theta_{1}\right)$ does not distinguish between these cases. Therefore it is not a fine moduli space and moreover it has a singularity.

There is a geometric way to treat these problems. One adds to the data $\left(M, \theta_{0}=0, \theta_{1}, t\right)$ a 'line'. This means the following. The assumption $\theta_{0}=0$ defines a lattice $\Lambda \subset \mathbb{C}((z)) \otimes M$ invariant under $\delta$. The 'line' is a 1-dimensional summand of $\Lambda$, invariant under $\delta$. We note that there are two cases:

(1) $\delta$ has matrix $\left(\begin{array}{ll}0 & 0 \\ 0 & 0\end{array}\right)$ on a basis $e_{1}, e_{2}$ of $\Lambda$. Then the possible lines are $\mathbb{C}[[z]] e$ with $e \in$ $\mathbb{C} e_{1}+\mathbb{C}_{2}, e \neq 0$. The possibilities form a $\mathbb{P}^{1}$ over $\mathbb{C}$.

(2) $\delta$ has matrix $\left(\begin{array}{ll}0 & 1 \\ 0 & 0\end{array}\right)$ on a basis $e_{1}, e_{2}$ of $\Lambda$. Then $\mathbb{C}[[z]] e_{1}$ is the only possible 'line'. 
This additional 'line' is called a "parabolic structure" (see $[4,6,7]$ ) or a "level structure". This defines a new set $\mathbf{S}^{+}\left(\theta_{0}=0, \theta_{1}\right)$ and a new moduli problem consisting of connections on the above $\mathcal{V}$ and an invariant line in $\mathcal{V}_{0} \otimes \mathbb{C}[[z]]$ (here $\mathcal{V}_{0}$ denotes the stalk of $\mathcal{V}$ at $z=0$ ). There is a fine moduli space which we call $\mathcal{M}^{+}\left(\theta_{0}=0, \theta_{1}\right)$. The natural morphism $\mathcal{M}^{+}\left(\theta_{0}=0, \theta_{1}\right) \rightarrow$ $\mathcal{M}\left(\theta_{0}=0, \theta_{1}\right)$ turns out to be the resolution of the latter space, seen as a surface over $\mathbb{C}(t)$ or as a surface after fixing a value for $t$. The preimage of the singular point is the projective line over $\mathbb{C}$. This construction is also present in the papers $[21,22]$ and we will not make it explicit here.

Something similar has to be done for the case $\theta_{0} \neq 0, \theta_{1}=0$ and the case $\theta_{0}=\theta_{1}=0$. In all cases we write $\mathcal{M}^{+}\left(\theta_{0}, \theta_{1}\right)$ for the moduli space obtained in this way and, for notational convenience we write $\mathcal{M}^{+}\left(\theta_{0}, \theta_{1}\right)=\mathcal{M}\left(\theta_{0}, \theta_{1}\right)$ also in the case $\theta_{0} \neq 0, \theta_{1} \neq 0$.

Observation 2.5. Consider a lattice $\Lambda$ over $\mathbb{C}[[z]]$ of rank two with a differential operator $\delta$ which has operator form $z \frac{d}{d z}+\left(\begin{array}{ll}a & c \\ b & d\end{array}\right)$ with $a, b, c, d \in \mathbb{C}[[z]]$ and $\left(\begin{array}{ll}a & c \\ b & d\end{array}\right) \equiv\left(\begin{array}{ll}\alpha & 0 \\ 0 & \beta\end{array}\right) \bmod (z)$. Suppose that $\beta-\alpha \notin \mathbb{Z}_{<0}$. Then $\Lambda$ has a unique direct summand $\mathbb{C}[[z]]$ e such that $\delta e=\alpha e$.

The proof is obtained by conjugating the operator $z \frac{d}{d z}+\left(\begin{array}{ll}a & c \\ b & d\end{array}\right)$ with a suitable invertible matrix $\left(\begin{array}{cc}t_{1} & 0 \\ t_{2} & t_{3}\end{array}\right) \in \mathrm{GL}_{2}(\mathbb{C}[[z]])$. After conjugation with a diagonal matrix in $\mathrm{GL}_{2}(\mathbb{C}[[z]])$ one can suppose that the differential operator is $z \frac{d}{d z}+\left(\begin{array}{cc}\alpha & c \\ b & \beta\end{array}\right)$ and $b, c \in z \mathbb{C}[[z]]$. There exists $x \in \mathbb{C}[[z]]$ such that $\left(\begin{array}{cc}1 & 0 \\ -x & 1\end{array}\right)\left\{z \frac{d}{d z}+\left(\begin{array}{cc}\alpha & c \\ b & \beta\end{array}\right)\right\}\left(\begin{array}{ll}1 & 0 \\ x & 1\end{array}\right)$ equals $z \frac{d}{d z}+\left(\begin{array}{cc}\alpha & * \\ 0 & \beta\end{array}\right)$. Indeed, this condition is equivalent to the equation $z \frac{d x}{d z}+(\beta-\alpha) x+b-c x^{2}=0$. Write $x=\sum_{n \geq 1} a_{n} z^{n}$. The equation reads

$$
\sum_{n \geq 1}(n+\beta-\alpha) a_{n} z^{n}+\sum_{n \geq 1} b_{n} z^{n}-\sum_{n \geq 1} c_{n} z^{n} \cdot\left(\sum_{n \geq 1} a_{n} z^{n}\right)^{2}=0
$$

and there is a unique solution.

Now we consider the moduli space $\mathcal{M}\left(\theta_{0}, \theta_{1}\right)$ for some $\theta_{0} \in \mathbb{Z}_{>0}$. From Observation 2.5 one concludes that the invariant lattice $\Lambda$ over $\mathbb{C}[[z]]$ has a direct summand $\mathbb{C}[[z]] e$ such that $\delta e=-\frac{\theta_{0}}{2} e$. In other words, there is a unique 'line' present in this situation and the 'level structure' at $z=0$ is already present. This explains why $\mathcal{M}\left(\theta_{0}, \theta_{1}\right)$ has no singularities for non zero integer values of $\theta_{0}$ and $\theta_{1}$.

Another interesting way to produce the correct moduli space for the cases $\theta_{0}=0$ and/or $\theta_{1}=0$ is the following. A differential module $M$ with the required data is represented by a connection on a vector bundle $\mathcal{V}$ of rank 2 , with degree -3 instead of -1 . Then $\mathcal{V}$ is identified with $O(-[\infty]) e_{1} \oplus O(-2[\infty]) e_{2}$. The connection $\nabla: \mathcal{V} \rightarrow \mathcal{V} \otimes \Omega([0]+[1]+2[\infty])$ is prescribed by the local differential operators

$$
z \frac{d}{d z}+\left(\begin{array}{cc}
\frac{\theta_{0}}{2} & 0 \\
0 & -\frac{\theta_{0}}{2}-1
\end{array}\right), \quad(z-1) \frac{d}{d z}+\left(\begin{array}{cc}
\frac{\theta_{1}}{2} & 0 \\
0 & -\frac{\theta_{1}}{2}-1
\end{array}\right), \quad z \frac{d}{d z}+\left(\begin{array}{cc}
-\frac{3}{4} & t^{2} z \\
1 & -\frac{1}{4}
\end{array}\right) .
$$

As in Section 2.3 one has to consider two affine parts. On the first part the operator reads

$$
\frac{d}{d z}+\frac{1}{z(z-1)}\left(\begin{array}{cc}
a_{0} & c_{0}+c_{1} z+c_{2} z^{2}+c_{3} z^{3} \\
z+b_{0} & d_{0}+d_{1} z+d_{2} z^{2}
\end{array}\right)
$$

and on the second part it is

$$
\frac{d}{d z}+\frac{1}{z(z-1)}\left(\begin{array}{cc}
a_{2} z^{2} & c_{0}+c_{1} z+c_{2} z^{2}+c_{3} z^{3} \\
1+b_{1} z & d_{0}+d_{1} z+d_{2} z^{2}
\end{array}\right) .
$$

The equations for the entries in these matrices are similar to those of Sections 2.3.1 and 2.3.2. After a computation one finds that singular points only occur for the cases $\theta_{0}=-1$ and/or 
$\theta_{1}=-1$. In particular, this produces a smooth moduli space for, say, $\theta_{0}=0$ and $\theta_{1} \neq-1$. Other choices for $\mathcal{V}$ with negative odd degree $-2 d-1$ and local equations at $z=0$ and $z=1$ where the above matrices have traces $-d,-d$ can be used to construct smooth moduli spaces for all combinations of $\theta_{0}$ and $\theta_{1}$.

In the sequel we write $\mathcal{M}^{+}\left(\theta_{0}, \theta_{1}\right)$ for the resolution of the space $\mathcal{M}\left(\theta_{0}, \theta_{1}\right)$. We note that $\mathcal{M}^{+}\left(\theta_{0}, \theta_{1}\right)$ is in general not yet the Okamoto-Painlevé space for the following reason. For any $a \in \mathbb{C}^{*}$, the closed subspace of $\mathcal{M}^{+}\left(\theta_{0}, \theta_{1}\right)$, given by $t=a$, is simply connected. This follows from Observation 2.4 and the fact that a fibre of $\mathcal{M}^{+}\left(\theta_{0}, \theta_{1}\right) \rightarrow \mathcal{M}\left(\theta_{0}, \theta_{1}\right)$ is either a point or a projective line over $\mathbb{C}$.

The 't-part' of $\mathcal{M}^{+}\left(\theta_{0}, \theta_{1}\right)$ runs in $\mathbb{C}^{*}$, which is not simply connected. In the earlier definition of Okamoto's space of initial values 'simply connected' was required. However, in one of the later papers of Okamoto et al. [10] the condition 'simply connected' for 'the space of initial values' is removed.

The other reason to replace $t$ by $e^{2 \pi i u}$ with $u \in \mathbb{C}$ is the following. The map from a tuple $\left(M, \theta_{0}, \theta_{1}, t\right)$ to the monodromy data at $z=\infty$ depends on the choice of a direction for multisummation. This direction has to be different from the singular direction and the latter moves with $t$.

As we will see in Section 4 , the monodromy space $\mathcal{R}\left(s_{0}, s_{1}\right)$ has for the values $s_{0}= \pm 2$ and $s_{1}= \pm 2$ singular points. Further $s_{0}=e^{\pi i \theta_{0}}+e^{-\pi i \theta_{0}}$ and $s_{1}=e^{\pi i \theta_{1}}+e^{-\pi i \theta_{1}}$. Also in this case one has to add a similar level structure as a method to obtain a desingularisation $\mathcal{R}^{+}\left(s_{0}, s_{1}\right)$ of $\mathcal{R}\left(s_{0}, s_{1}\right)$. Further we will show that $\mathcal{R}^{+}\left(s_{0}, s_{1}\right)$ is simply connected.

\section{Computation of the Painlevé equation $\operatorname{deg} \mathrm{P}_{\mathrm{V}}$}

This calculation is done on the first affine part of the space $\mathcal{M}\left(\theta_{0}, \theta_{1}\right)$. There an isomonodromic family $\frac{d}{d z}+A$ with $A=A(z, t)$ is given and computed by the assumption that this operator commutes with an unknown operator $\frac{d}{d t}+B$ with $B=B(z, t)$. This is equivalent to the formula $\frac{\partial A}{\partial t}=\frac{\partial B}{\partial z}+[A, B]$

From the singularities of $A$ one derives that $B$ is w.r.t. $z$ a polynomial matrix of degree at most 1 . Further, both $A$ and $B$ are $2 \times 2$-matrices with trace 0 . This we use to make the computations smoother.

Write $H=\left(\begin{array}{cc}1 & 0 \\ 0 & -1\end{array}\right), E_{1}=\left(\begin{array}{ll}0 & 1 \\ 0 & 0\end{array}\right), E_{2}=\left(\begin{array}{ll}0 & 0 \\ 1 & 0\end{array}\right)$. Observe $\left[H, E_{1}\right]=2 E_{1},\left[H, E_{2}\right]=-2 E_{2}$, $\left[E_{1}, E_{2}\right]=H$. In the sequel we write $f^{\prime}$ for $\frac{d f}{d t}$. Write $A=\frac{a_{0}}{z(z-1)} H+\frac{c_{0}+c_{1} z+t^{2} z^{2}}{z(z-1)} E_{1}+\frac{z+b_{0}}{z(z-1)} E_{2}$. Write $B=B_{H} H+B_{1} E_{1}+B_{2} E_{2}$ and $B_{H}=B_{H, 0}+B_{H, 1} z, B_{1}=B_{1,0}+B_{1,1} z, B_{2}=B_{2,0}+B_{2,1} z$ where the $B_{*, *}$ only depend on $t$. The equation $\frac{\partial A}{\partial t}=\frac{\partial B}{\partial z}+[A, B]$, multiplied by $z(z-1)$, has coefficients with respect to the basis $H, E_{1}, E_{2}$ which read:

$$
\begin{aligned}
(H) \quad a_{0}^{\prime}=z(z-1) B_{H, 1}+\left(c_{0}+c_{1} z+t^{2} z^{2}\right)\left(B_{2,0}+B_{2,1} z\right)-\left(z+b_{0}\right)\left(B_{1,0}+z B_{1,1}\right), & \left(E_{1}\right) \quad c_{0}^{\prime}+c_{1}^{\prime} z+2 t z^{2}=z(z-1) B_{1,1}+2 a_{0}\left(B_{1,0}+B_{1,1} z\right) \\
& \quad-2\left(B_{H, 0}+B_{H, 1} z\right)\left(c_{0}+c_{1} z+t^{2} z^{2}\right), \\
\left(E_{2}\right) & b_{0}^{\prime}=z(z-1) B_{2,1}-2 a_{0}\left(B_{2,0}+B_{2,1} z\right)+2\left(B_{H, 0}+B_{H, 1} z\right)\left(z+b_{0}\right) .
\end{aligned}
$$

Each of these three equations is considered with respect to the degrees in $z$. A sequence of solving equations (in a suitable order!) yields the following:

$(H)$ degree 3 implies $B_{2,1}=0$; degree 2 implies $0=B_{H, 1}+t^{2} B_{2,0}-B_{1,1}$ and so $B_{2,0}=2 t^{-1}$; degree 1 implies $0=c_{1} B_{2,0}-B_{1,0}-b_{0} B_{1,1}$ and so $B_{1,0}=c_{1} \cdot 2 t^{-1}-b_{0} \cdot 2 t$; degree 0 implies $a_{0}^{\prime}=c_{0} B_{2,0}-b_{0} B_{1,0}$ and so $a_{0}^{\prime}=2 t^{-1} c_{0}-b_{0}\left(2 t^{-1} c_{1}-2 t b_{0}\right)$; 
$\left(E_{1}\right)$ degree 3 implies $B_{H, 1}=0$; degree 2 implies $2 t=B_{1,1}-2 B_{H, 0} t^{2}$ and so $B_{1,1}=2 t$; degree 1 implies $c_{1}^{\prime}=-B_{1,1}+2 a_{0} B_{1,1}$ and so $c_{1}^{\prime}=2 t\left(2 a_{0}-1\right)$; degree 0 implies $c_{0}^{\prime}=2 a_{0} B_{1,0}$ and so $c_{0}^{\prime}=2 a_{0}\left(c_{1} \cdot 2 t^{-1}-b_{0} \cdot 2 t\right)$;

$\left(E_{2}\right) b_{0}^{\prime}=-2 a_{0} B_{2,0}+2 B_{H, 0}\left(z+b_{0}\right)$ and thus $B_{H, 0}=0$ and $b_{0}^{\prime}=-2 a_{0} B_{2,0}$ and so $b_{0}^{\prime}=-2 a_{0} \cdot 2 t^{-1}$.

This leads to the following set of equations:

$$
\begin{aligned}
& \text { (1) } a_{0}^{2}+b_{0} c_{0}=\frac{\theta_{0}^{2}}{4}, \\
& \text { (2) } c_{0}=-\frac{\theta_{0}^{2}}{4}+\frac{\theta_{1}^{2}}{4}-c_{1}-t^{2}-b_{0} c_{1}-b_{0} t^{2}, \\
& \text { (3) } a_{0}^{\prime}=2 t^{-1} c_{0}-b_{0}\left(2 t^{-1} c_{1}-2 t b_{0}\right), \\
& \text { (4) } c_{1}^{\prime}=-2 t+4 a_{0} t \\
& \text { (5) } c_{0}^{\prime}=2 a_{0}\left(2 t^{-1} c_{1}-2 t b_{0}\right), \\
& \text { (6) } b_{0}^{\prime}=-4 a_{0} t^{-1} .
\end{aligned}
$$

This is solved, using MAPLE, by the following steps: Eliminate $c_{0}$ by (2) and $a_{0}$ by using (6). In the new set of equations one can eliminate $c_{1}$ in a linear way. Then MAPLE yields a secondorder equation for $b_{0}$. Write the matrix differential operator as $\frac{d}{d z}+\left(\begin{array}{cc}a & c \\ b & -a\end{array}\right)$. Then taking the first vector as cyclic vector one obtains a scalar equation $\left(\frac{d}{d z}\right)^{2}-\frac{c^{\prime}}{c} \frac{d}{d z}-a^{\prime}-a^{2}-b c+a \frac{c^{\prime}}{c}$. The $q$ for the Painlevé equation is the pole $\neq 0,1, \infty$ of this scalar equation. Thus $q=-b_{0}$. The Hamiltonian system for the Painlevé equation has variables $q$ and $p$ where $p$ is the residue of the term $-a^{\prime}-a^{2}-b c+a \frac{c^{\prime}}{c}$ at $z=q$. One finds in this way for $\operatorname{deg}_{\mathrm{V}}$ and the Hamiltonian function $\mathcal{H}$ the formulas

$$
\begin{aligned}
& q^{\prime \prime}=\frac{1}{2}\left(\frac{1}{q}+\frac{1}{q-1}\right)\left(q^{\prime}\right)^{2}-\frac{q^{\prime}}{t}+\frac{2(q-1) \theta_{0}^{2}}{q t^{2}}-\frac{2 q \theta_{1}^{2}}{(q-1) t^{2}}+8 q(q-1), \\
& p=\frac{t}{4} q^{\prime}, \quad \mathcal{H}=\frac{2\left(p^{2}-\frac{\theta_{0}^{2}}{4}\right)}{t q}-\frac{2\left(p^{2}-\frac{\theta_{1}^{2}}{4}\right)}{t(q-1)}+2 q t,
\end{aligned}
$$

with here, exceptionally, $q^{\prime}=q(1-q) \frac{\partial \mathcal{H}}{\partial p}$ and $p^{\prime}=-q(1-q) \frac{\partial \mathcal{H}}{\partial q}$.

We note that the formula for $\operatorname{deg} \mathrm{P}_{\mathrm{V}}$ coincides with the one in [19].

The relation between this $\operatorname{deg} \mathrm{P}_{\mathrm{V}}$ and the classical degenerate $\mathrm{P}_{\mathrm{V}}$ is the following. Consider solutions $q(t)$ of $\operatorname{deg}_{\mathrm{V}}$ which are even. Then these are written as $q(t)=Q\left(t^{2}\right)$ for some function $Q(s)$. One easily computes that the second-order differential equation for $Q$ is

$$
Q^{\prime \prime}=\frac{1}{2}\left(\frac{1}{Q}+\frac{1}{Q-1}\right)\left(Q^{\prime}\right)^{2}-\frac{Q^{\prime}}{s}+\frac{(Q-1) \frac{\theta_{o}^{2}}{2}}{Q s^{2}}-\frac{Q \frac{\theta_{1}^{2}}{2}}{(Q-1) s^{2}}+\frac{2 Q(Q-1)}{s} .
$$

One substitutes $Q=\frac{y}{y-1}$ and finds for $y$ the second-order differential equation

$$
y^{\prime \prime}=\frac{1}{2} \frac{3 y-1}{y(y-1)}\left(y^{\prime}\right)^{2}-\frac{y^{\prime}}{s}+\frac{y(y-1)^{2} \theta_{1}^{2}}{2 s^{2}}-\frac{(y-1)^{2} \theta_{0}^{2}}{2 s^{2} y}-\frac{2 y}{s} .
$$

This is the classical degenerate $\mathrm{P}_{\mathrm{V}}(\alpha, \beta, \gamma, \delta)$, normalized as the following special case $\mathrm{P}_{\mathrm{V}}\left(\frac{\theta_{1}^{2}}{2}\right.$, $\left.-\frac{\theta_{0}^{2}}{2},-2,0\right)$ of $\mathrm{P}_{\mathrm{V}}$.

Remark 3.1. The formula for $\mathrm{P}_{\mathrm{V}}$ in [19] reduces to the classical formula for $\mathrm{P}_{\mathrm{V}}$ as well, using the same substitution $q=\frac{y}{y-1}$. 


\section{The moduli space for the analytic data $\mathcal{R}\left(s_{0}, s_{1}\right)$}

We reproduce here, with a slightly different choice of signs, the paper [19]. The solution space $V$ at $z=\infty$ has a basis $e_{1}, e_{2}$ such that the formal monodromy is $\left(\begin{array}{cc}0 & -1 \\ 1 & 0\end{array}\right)$ and the only Stokes matrix has the matrix $\left(\begin{array}{ll}1 & 0 \\ e & 1\end{array}\right)$. The topological monodromy at $z=\infty$ is the product $M_{\infty}=\left(\begin{array}{cc}-e & -1 \\ 1 & 0\end{array}\right)$. By multisummation in a suitable direction we combine this with the monodromy matrices $M_{0}$ and $M_{1}$ for loops around $z=0$ and $z=1$. Put $s_{0}=e^{\pi i \theta_{0}}+e^{-\pi i \theta_{0}}, s_{1}=e^{\pi i \theta_{1}}+e^{-\pi i \theta_{1}}$. Then $M_{0}, M_{1}$ have determinants 1 and traces $s_{0}, s_{1}$. One has the relation $M_{0} M_{1} M_{\infty}=1$.

The basis $e_{1}, e_{2}$ is unique up to $e_{1}, e_{2} \mapsto \lambda e_{1}, \lambda e_{2}$. This transformation acts trivially on the matrices. Write $M_{1}=\left(\begin{array}{ll}a_{1} & b_{1} \\ c_{1} & d_{1}\end{array}\right)$. Then $M_{0}$ is the inverse of $M_{1} M_{\infty}$. Hence $\mathcal{R}\left(s_{0}, s_{1}\right)$ is the affine space with coordinate ring $\mathbb{C}\left[a_{1}, b_{1}, c_{1}, d_{1}, e\right]$ with the relations

$$
a_{1} d_{1}-b_{1} c_{1}=1, \quad a_{1}+d_{1}=s_{1}, \quad-a_{1} e+b_{1}-c_{1}=s_{0} .
$$

Elimination of $c_{1}, d_{1}$ and $x_{1}=-b_{1}, x_{2}=-a_{1}, x_{3}=-e$ leads to the ring $\mathbb{C}\left[x_{1}, x_{2}, x_{3}\right] /\left(x_{1} x_{2} x_{3}+\right.$ $\left.x_{1}^{2}+x_{2}^{2}+s_{0} x_{1}+s_{1} x_{2}+1\right)$.

Only for $s_{1}= \pm 2$ and for $s_{0}= \pm 2$ the corresponding affine cubic surface $\mathcal{R}\left(s_{0}, s_{1}\right)$ has singularities:

$$
\begin{aligned}
& s_{1}= \pm 2, \quad x_{1}=0, \quad x_{2}=\mp 1, \quad x_{3}= \pm s_{0}, \quad \text { and } \\
& s_{0}= \pm 2, \quad x_{1}=\mp 1, \quad x_{2}=0, \quad x_{3}= \pm s_{1} \text {. }
\end{aligned}
$$

In particular for $s_{0} \neq \pm 2, s_{1} \neq \pm 2$ the space $\mathcal{R}\left(s_{0}, s_{1}\right)$ has no singularities.

Observation 4.1. $\mathcal{R}\left(s_{0}, s_{1}\right)$ is simply connected for all $s_{0}, s_{1}$.

Proof. Consider the projection of $\mathcal{R}\left(s_{0}, s_{1}\right) \rightarrow \mathbb{C}^{2}$, by $\left(x_{1}, x_{2}, x_{3}\right) \mapsto\left(x_{1}, x_{2}\right)$. The fibres of this map are either a point or $\mathbb{C}$ or empty. The image $B$ is the union of $\left(\mathbb{C}^{*}\right)^{2}$ with the points $\left\{\left(0, x_{2}\right) \mid x_{2}^{2}+s_{1} x_{2}+1=0\right\}$ and $\left\{\left(x_{1}, 0\right) \mid x_{1}^{2}+s_{0} x_{1}+1=0\right\}$. It suffices to show that $B$ is simply connected. The inclusion $\left(\mathbb{C}^{*}\right)^{2} \subset B$ induces a surjection $\pi_{1}\left(\left(\mathbb{C}^{*}\right)^{2}, *\right) \rightarrow \pi_{1}(B, *)$. Consider a generator of $\pi_{1}\left(\left(\mathbb{C}^{*}\right)^{2}, *\right)$, represented by the loop $s \in[0,1] \mapsto\left(\tilde{x}_{1}, e^{2 \pi i s}\right)$, where $\tilde{x}_{1}$ is chosen such that $\tilde{x}_{1}^{2}+s_{0} \tilde{x}_{1}+1=0$. In $B$ this loop is homotopic to the constant loop $s \mapsto\left(\tilde{x}_{1}, 0\right)$ by the homotopy $(s, \lambda) \in[0,1]^{2} \mapsto\left(\tilde{x}_{1}, \lambda e^{2 \pi i s}\right)$. One concludes that the two generators of $\pi_{1}\left(\left(\mathbb{C}^{*}\right)^{2}, *\right)$ have trivial image in $\pi_{1}(B, *)$ and that $\pi_{1}(B, *)=1$.

As in Section 2.3.3, the geometric way to resolve the singularities of $\mathcal{R}\left(s_{0}, s_{1}\right)$ for $s_{0}= \pm 2$ and/or $s_{1}= \pm 2$ is to add a level structure consisting of a line (or two lines if both $s_{0}= \pm 2$ and $\left.s_{1}= \pm 2\right)$. The resulting space is denoted by $\mathcal{R}^{+}\left(s_{0}, s_{1}\right)$. We will work out the details for $s_{0} \neq \pm 2$ and $s_{1}=2$.

The fibre of the surjective morphism $\mathcal{R}^{+}\left(s_{0}, s_{1}\right) \rightarrow \mathcal{R}\left(s_{0}, s_{1}\right)$ is in general a point and there are at most two fibres isomorphic to $\mathbb{P}^{1}$. It follows that $\mathcal{R}^{+}\left(s_{0}, s_{1}\right)$ is simply connected as well.

For the formulation of the (extended) Riemann-Hilbert morphism we need to replace the space $T=\mathbb{C}^{*}$ of the variable $t$ by its universal covering $\tilde{T}=\mathbb{C}$. The reason is that the singular direction at infinity varies with $t$. Then

$$
\mathrm{RH}^{+}: \mathcal{M}^{+}\left(\theta_{0}, \theta_{1}\right) \times_{T} \tilde{T} \rightarrow \mathcal{R}^{+}\left(s_{0}, s_{1}\right) \times \tilde{T}
$$

is a well defined analytic map. This (extended) Riemann-Hilbert map $\mathrm{RH}^{+}$is bijective on points. Indeed, the points on the left hand side correspond to the tuples $\left(M, \theta_{0}, \theta_{1}, t\right)$ with additionally $u \in \mathbb{C}$ with $t=e^{2 \pi i u}$ and level structure(s) if needed. The points on the right hand side correspond to the analytic data with level structure (if needed), $u \in \mathbb{C}$ and the formal structure at $z=\infty$. By [19, Theorem 1.7], these two sets coincide. As in [18, Theorem 1.5] we conclude that $\mathrm{RH}^{+}$is an analytic isomorphism between two algebraic varieties over $\mathbb{C}$. Moreover, as in the proof of loc. sit., from the isomorphism one obtains (compare [10]) 
Theorem 4.2. The Painlevé property for $\operatorname{deg}_{\mathrm{V}}\left(\theta_{0}, \theta_{1}\right)$ holds. Moreover $\mathcal{M}^{+}\left(\theta_{0}, \theta_{1}\right) \times_{T} \tilde{T}$ is the Okamoto-Painlevé space.

The resolution $\mathcal{R}^{+}\left(s_{0}, 2\right) \rightarrow \mathcal{R}\left(s_{0}, 2\right)$ for $s_{0} \neq \pm 2$. As before, any differential module $M$ (with the given data) determines a basis $e_{1}, e_{2}$ of the solution space $V$ at $z=\infty$ such that the formal monodromy $\gamma$ has matrix $\left(\begin{array}{cc}0 & -1 \\ 1 & 0\end{array}\right)$. This basis is unique up to multiplication of $e_{1}, e_{2}$ by the same constant. All maps are written as matrices with respect to this basis.

A point of $\mathcal{R}^{+}\left(s_{0}, 2\right)$ corresponds to a tuple $\left(M_{0}, M_{1}, M_{\infty}, \mathbb{C} v\right)$ where $M_{0}, M_{1}, M_{\infty}$ are the matrices for the topological monodromies for the points $0,1, \infty$. Then $M_{0} M_{1} M_{\infty}=1$ and $v$ is a non zero eigenvector of $M_{1}$ (for the eigenvalue 1). Write $M_{1}=\left(\begin{array}{cc}a_{1} & b_{1} \\ c_{1} & d_{1}\end{array}\right), M_{\infty}=\left(\begin{array}{cc}-e & -1 \\ 1 & 0\end{array}\right)$, $v=\left(\begin{array}{l}y_{0} \\ y_{1}\end{array}\right)$. There are two affine parts given by the cases $y_{0} \neq 0$ and $y_{1} \neq 0$. We consider here the first case and normalize $y_{0}=1$.

The matrix $M_{0}$ is determined by $M_{0} M_{1} M_{\infty}=1$. The equations in the variables $a_{1}, b_{1}, c_{1}$, $d_{1}, e, y_{1}$ are

$$
a_{1}+d_{1}=2, \quad a_{1} d_{1}-b_{1} c_{1}=1, \quad a_{1}-1+b_{1} y_{1}=0, \quad c_{1}+\left(d_{1}-1\right) y_{1}=0,
$$

where the last two equations come from: $v$ is eigenvector for $M_{1}$. One eliminates $c_{1}, d_{1}$ by $a_{1}+d_{1}=2$ and $-a_{1} e+b_{1}-c_{1}=s_{0}$. One writes $x_{1}=-b_{1}, x_{2}=-a_{1}, x_{3}=-e$. Thus the ring of regular functions on the affine part that we are looking at has the form $\mathbb{C}\left[x_{1}, x_{2}, x_{3}, y_{1}\right] /$ relations and the relations are

$$
\begin{aligned}
& x_{1} x_{2} x_{3}+x_{1}^{2}+x_{2}^{2}+s_{0} x_{1}+2 x_{2}+1=0, \quad\left(x_{2}+1\right)+x_{1} y_{1}=0, \\
& \left(1+x_{2}\right) y_{1}=x_{2} x_{3}+x_{1}+s_{0} .
\end{aligned}
$$

The morphism $\mathcal{R}^{+}\left(s_{0}, 2\right) \rightarrow \mathcal{R}\left(s_{0}, 2\right)$, restricted to this affine part, is given by the obvious homomorphism

$$
\mathbb{C}\left[x_{1}, x_{2}, x_{3}\right] /\left(x_{1} x_{2} x_{3}+x_{1}^{2}+x_{2}^{2}+s_{0} x_{1}+2 x_{2}+1\right) \rightarrow \mathbb{C}\left[x_{1}, x_{2}, x_{3}, y_{1}\right] / \text { relations. }
$$

For $x_{1} \neq 0$ one can eliminate $y_{1}$ by using the equation $\left(x_{2}+1\right)+x_{1} y_{1}=0$. In fact, the above map is an isomorphism after inverting the element $x_{1}$. For $x_{1}=0$ one finds that $\left(x_{1}, x_{2}, x_{3}\right)=$ $\left(0,-1, s_{0}\right)$ and this is the unique singular point of $\mathcal{R}\left(s_{0}, 2\right)$. The points lying above this singular point are $\left(x_{1}, x_{2}, x_{3}, y_{1}\right)=\left(0,-1, s_{0}, a\right)$ for all $a \in \mathbb{C}$. One easily verifies that these points of $\mathcal{R}^{+}\left(s_{0}, 2\right)$ are smooth.

The computation of the affine part $y_{1} \neq 0$ is similar. One concludes that $\mathcal{R}^{+}\left(s_{0}, 2\right) \rightarrow \mathcal{R}\left(s_{0}, 2\right)$ is a resolution of singularities and that the fibre above the singular point is $\mathbb{P}^{1}$.

\section{Computing the Bäcklund transformations}

Write $\mathbf{S}$ for the union of the sets $\mathbf{S}\left(\theta_{0}, \theta_{1}\right)$ taken over all $\theta_{0}, \theta_{1}$. We recall that $\theta_{0}, \theta_{1}, t$ determine the invariant lattices at $z=0,1, \infty$. A 'natural' automorphism of $\mathbf{S}$ may change a given tuple $\left(M, \theta_{0}, \theta_{1}, t\right)$ into the same module $M$ but with different lattices. For example, the lattice at $z=0$ will be changed by replacing $\theta_{0}$ by $\theta_{0}+2$. Further the module $M$ can be changed and one can consider the automorphism of $\mathbb{P}^{1}$ which interchanges $z=0,1$ and has $z=\infty$ as fixed point.

Some 'natural' automorphisms of $\mathbf{S}$ are given by the tuple $\left(M, \theta_{0}, \theta_{1}, t\right) \mapsto$

(1) $\left(M, \theta_{0}, \theta_{1},-t\right)$,

(2) $\left(M,-\theta_{0}, \theta_{1}, t\right)$

(3) $\left(M, \theta_{0},-\theta_{1}, t\right)$, 
(4) $\left(\tilde{M}, \theta_{1}, \theta_{0}\right)$ where $\tilde{M}$ is obtained from $M$ by the automorphism $z \mapsto 1-z$,

(5) $\left(N \otimes M, \theta_{0}+1, \theta_{1}, t\right)$ where $N$ is the 1 -dimensional module represented by $\frac{d}{d z}+\frac{1}{2 z}$.

These special automorphisms will be lifted to isomorphisms between various moduli spaces $\mathcal{M}^{+}\left(\theta_{0}, \theta_{1}\right)$. The isomorphisms preserve the analytic data and map therefore solutions of one $\operatorname{deg} \mathrm{P}_{\mathrm{V}}$ equation to solutions of another. They are Bäcklund transformations for the $\operatorname{deg} \mathrm{P}_{\mathrm{V}}$ and we hope that we found all of them in this way.

Discussion of the transformations: (1) only changes the variable. It induces the Bäcklund transformation which sends a solution $q(t)$ of $\operatorname{deg} \mathrm{P}_{\mathrm{V}}\left(\theta_{0}, \theta_{1}\right)$ to another solution $q(-t)$ of that equation. (2) and (3) induce the identity on the solutions of all $\operatorname{deg} \mathrm{P}_{\mathrm{V}}$.

(4) Consider a point $M$ of $\mathcal{M}\left(\theta_{0}, \theta_{1}\right)$ belonging to the first affine chart of this variety. It is represented by $\frac{d}{d z}+\frac{1}{z(z-1)}\left(\begin{array}{c}a_{0} \\ z-q \\ c_{0}+c_{1} z+t^{2} z^{2} \\ -a_{0}\end{array}\right)$. The change $z \mapsto 1-z$ applied to this operator yields

$$
\frac{d}{d z}+\frac{1}{z(z-1)}\left(\begin{array}{cc}
-a_{0} & -c_{0}-c_{1}(1-z)-t^{2}(1-z)^{2} \\
z+q-1 & a_{0}
\end{array}\right) .
$$

A small computation shows that after changing $t$ into it this becomes a differential operator belonging to $\mathcal{M}\left(\theta_{1}, \theta_{0}\right)$. This produces the Bäcklund transformation $q(t) \mapsto-q(i t)+1$ which sends a solution of $\operatorname{deg} \mathrm{P}_{\mathrm{V}}\left(\theta_{0}, \theta_{1}\right)$ to a solution of $\operatorname{deg} \mathrm{P}_{\mathrm{V}}\left(\theta_{1}, \theta_{0}\right)$.

(5) $M$ is locally represented by $z \frac{d}{d z}+\left(\begin{array}{cc}\omega_{0} & 0 \\ 0 & -\omega_{0}\end{array}\right)$ with $\omega_{0}=\frac{\theta_{0}}{2}$ and by $z \frac{d}{d z}+\left(\begin{array}{cc}\frac{-3}{4} & t^{2} z \\ 1 & -\frac{1}{4}\end{array}\right)$. After taking the tensor product with $N$ these operators become $z \frac{d}{d z}+\left(\begin{array}{cc}\omega_{0}+\frac{1}{2} & 0 \\ 0 & -\omega_{0}+\frac{1}{2}\end{array}\right)$ and $z \frac{d}{d z}+\left(\begin{array}{cc}\frac{-3}{4}+\frac{1}{2} & t^{2} z \\ 1 & -\frac{1}{4}+\frac{1}{2}\end{array}\right)$. By multiplying one basis vector by $z^{-1}$, the first operator becomes $z \frac{d}{d z}+\left(\begin{array}{cc}\omega_{0}+\frac{1}{2} & 0 \\ 0 & -\omega_{0}-\frac{1}{2}\end{array}\right)$ and the second becomes $z \frac{d}{d z}+\left(\begin{array}{cc}\frac{-3}{4}-\frac{1}{2} & t^{2} z \\ 1 & -\frac{1}{4}+\frac{1}{2}\end{array}\right)$. This shows the validity of (5).

Using MAPLE one can compute the actual isomorphism between the two moduli spaces. In terms of matrix differential operators, this works as follows. Let the differential operator $\frac{d}{d z}+A(a, q)$ represent an open part of the first affine chart $\mathcal{M}_{1}\left(\theta_{0}, \theta_{1}\right)$. Here $q$ stands for $-b_{0}$ (as before) and $a$ denotes $a_{0}$. Further $c_{1}$ is written as a rational function in $a$ and $q$.

Similarly, let $\frac{d}{d z}+\tilde{A}(\tilde{a}, \tilde{q})$ denote the differential operator on an open part of $\mathcal{M}\left(\theta_{0}+1, \theta_{1}\right)$. Then there exists $U \in \mathrm{GL}_{2}(\mathbb{C}(z))$ such that

$$
U\left(\frac{d}{d z}+A(a, q)+\left(\begin{array}{cc}
\frac{1}{2 z} & 0 \\
0 & \frac{1}{2 z}
\end{array}\right)\right) U^{-1}=\frac{d}{d z}+\tilde{A}(\tilde{a}, \tilde{q}) .
$$

Further $U, U^{-1}$ are seen to have poles of order $\leq 1$ at $z=0$ and $z=\infty$ and no further poles. This information suffices for the computation of the solution $\tilde{q}$ of $\operatorname{deg} \mathrm{P}_{\mathrm{V}}\left(\theta_{0}+1, \theta_{1}\right)$ in terms of the solution $q$ of $\operatorname{deg}_{\mathrm{V}}\left(\theta_{0}, \theta_{1}\right)$ and its first derivative (and $\left.t, \theta_{0}, \theta_{1}\right)$

$$
\tilde{q}=1-\frac{\theta_{0}^{2}(q-1)}{4 q^{2} t^{2}}+\frac{a \theta_{0}}{q^{2} t^{2}}+\frac{\theta_{1}^{2}}{4 t^{2}(q-1)}
$$

and a longer formula for $\tilde{a}$, namely

$$
\begin{aligned}
& \frac{q-1}{8 q^{3} t^{2}} \theta_{0}^{3}+\frac{q^{2}+2 a q-q-6 a}{8 q^{3} t^{2}} \theta_{0}^{2}+\frac{q-1}{2 q} \theta_{0}-\frac{a\left(q^{2}+2 a q-q-3 a\right)}{2(q-1) q^{3} t^{2}} \theta_{0}-\frac{\theta_{0} \theta_{1}^{2}}{8 q t^{2}(q-1)} \\
& \quad-\frac{a}{q}-\frac{q+2 a}{8 q(q-1) t^{2}} \theta_{1}^{2}+\frac{a^{2}(2 a+q)}{q^{3} t^{2}(q-1)} .
\end{aligned}
$$


Now we compare the group of the Bäcklund transformations for $\operatorname{deg}_{\mathrm{V}}$ with the work of N.S. Witte [26]. We restrict our transformations to the case of even solutions of $\operatorname{deg} \mathrm{P}_{\mathrm{V}}$ and find the classical $\mathrm{P}_{\mathrm{V}}\left(\frac{\theta_{1}^{2}}{2},-\frac{\theta_{0}^{2}}{2},-2,0\right)$. The formulas (21), (22), (23) of [26] lead to $v_{1}=\theta_{0}+\theta_{1}$ and $v_{2}=\theta_{0}-\theta_{1}$. Our group of transformations for the even solutions of $\operatorname{deg} \mathrm{P}_{\mathrm{V}}$ is generated by $\left(\theta_{0}, \theta_{1}\right) \mapsto\left(\theta_{0}+1, \theta_{1}\right)$ and $\left(\theta_{0}, \theta_{1}\right) \mapsto\left(\theta_{1}, \theta_{0}\right)$ and the 'trivial' transformations $\left(\theta_{0}, \theta_{1}\right) \rightarrow$ $\left( \pm \theta_{0}, \pm \theta_{1}\right)$. One easily verifies that this coincides with the group of transformations in [26].

We remark that the transformations $\theta_{0} \mapsto-\theta_{0}$ and/or $\theta_{1} \mapsto-\theta_{1}$ act trivially on solutions of $\operatorname{deg} \mathrm{P}_{\mathrm{V}}$. However (compare [26]), they do not act as the identity on a suitable Hamiltonian system for $\operatorname{deg} \mathrm{P}_{\mathrm{V}}$.

\section{Acknowledgments}

The authors thank Yousuke Ohyama for his many helpful answers to our questions, and the referees for their careful reading and useful suggestions. The first author thanks the Johann Bernoulli Institute of the University of Groningen and the Universidad Simón Bolívar for financial support to participate in this project.

\section{References}

[1] Chekhov L., Mazzocco M., Rubtsov V., Painlevé monodromy manifolds, decorated character varieties and cluster algebras, Int. Math. Res. Not., to appear, arXiv:1511.03851.

[2] Fokas A.S., Its A.R., Kapaev A.A., Novokshenov V.Yu., Painlevé transcendents. The Riemann-Hilbert approach, Mathematical Surveys and Monographs, Vol. 128, Amer. Math. Soc., Providence, RI, 2006.

[3] Gromak V.I., On the theory of Painlevé's equations, Differential Equations 11 (1975), 285-287.

[4] Inaba M., Moduli of parabolic connections on curves and the Riemann-Hilbert correspondence, J. Algebraic Geom. 22 (2013), 407-480, math.AG/0602004.

[5] Inaba M., Iwasaki K., Saito M.-H., Dynamics of the sixth Painlevé equation, in Théories asymptotiques et équations de Painlevé, Sémin. Congr., Vol. 14, Soc. Math. France, Paris, 2006, 103-167.

[6] Inaba M., Iwasaki K., Saito M.-H., Moduli of stable parabolic connections, Riemann-Hilbert correspondence and geometry of Painlevé equation of type VI. I, Publ. Res. Inst. Math. Sci. 42 (2006), 987-1089, math.AG/0309342.

[7] Inaba M., Iwasaki K., Saito M.-H., Moduli of stable parabolic connections, Riemann-Hilbert correspondence and geometry of Painlevé equation of type VI. II, in Moduli Spaces and Arithmetic Geometry, Adv. Stud. Pure Math., Vol. 45, Math. Soc. Japan, Tokyo, 2006, 387-432, math.AG/0605025.

[8] Jimbo M., Miwa T., Ueno K., Monodromy preserving deformation of linear ordinary differential equations with rational coefficients. I. General theory and $\tau$-function, Phys. D 2 (1981), 306-352.

[9] Jimbo M., Miwa T., Monodromy preserving deformation of linear ordinary differential equations with rational coefficients. II, Phys. D 2 (1981), 407-448.

[10] Ohyama Y., Kawamuko H., Sakai H., Okamoto K., Studies on the Painlevé equations. V. Third Painlevé equations of special type $\mathrm{P}_{\mathrm{III}}\left(D_{7}\right)$ and $\mathrm{P}_{\mathrm{III}}\left(D_{8}\right)$, J. Math. Sci. Univ. Tokyo 13 (2006), 145-204.

[11] Ohyama Y., Okumura S., R. Fuchs' problem of the Painlevé equations from the first to the fifth, in Algebraic and Geometric Aspects of Integrable Systems and Random Matrices, Contemp. Math., Vol. 593, Amer. Math. Soc., Providence, RI, 2013, 163-178, math.CA/0512243.

[12] Okamoto K., Sur les feuilletages associés aux équations du second ordre à points critiques fixes de P. Painlevé. Espaces des conditions initiales, Japan. J. Math. 5 (1979), 1-79.

[13] Okamoto K., Isomonodromic deformation and Painlevé equations, and the Garnier system, J. Fac. Sci. Univ. Tokyo Sect. IA Math. 33 (1986), 575-618.

[14] Okamoto K., Studies on the Painlevé equations. III. Second and fourth Painlevé equations, $\mathrm{P}_{\mathrm{II}}$ and $\mathrm{P}_{\mathrm{IV}}$, Math. Ann. 275 (1986), 221-255.

[15] Okamoto K., Studies on the Painlevé equations. IV. Third Painlevé equation $\mathrm{P}_{\text {III }}$, Funkcial. Ekvac. 30 (1987), 305-332. 
[16] Okamoto K., The Hamiltonians associated to the Painlevé equations, in The Painlevé property, CRM Ser. Math. Phys., Springer, New York, 1999, 735-787.

[17] van der Put M., Families of linear differential equations related to the second Painlevé equation, in Algebraic Methods in Dynamical Systems, Banach Center Publ., Vol. 94, Polish Acad. Sci. Inst. Math., Warsaw, 2011, $247-262$.

[18] van der Put M., Families of linear differential equations and the Painlevé equations, in Geometric and Differential Galois Theories, Sémin. Congr., Vol. 27, Soc. Math. France, Paris, 2013, 207-224.

[19] van der Put M., Saito M.-H., Moduli spaces for linear differential equations and the Painlevé equations, Ann. Inst. Fourier (Grenoble) 59 (2009), 2611-2667, arXiv:0902.1702.

[20] van der Put M., Singer M.F., Galois theory of linear differential equations, Grundlehren der Mathematischen Wissenschaften, Vol. 328, Springer-Verlag, Berlin, 2003.

[21] van der Put M., Top J., A Riemann-Hilbert approach to Painlevé IV, J. Nonlinear Math. Phys. 20 (2013), suppl. 1, 165-177, arXiv:1207.4335.

[22] van der Put M., Top J., Geometric aspects of the Painlevé equations PIII(D $\left.D_{6}\right)$ and PIII(D $)$, SIGMA 10 (2014), 050, 24 pages, arXiv:1207.4023.

[23] Saito M.-H., Takebe T., Classification of Okamoto-Painlevé pairs, Kobe J. Math. 19 (2002), 21-50, math.AG/0006028.

[24] Saito M.-H., Takebe T., Terajima H., Deformation of Okamoto-Painlevé pairs and Painlevé equations, J. Algebraic Geom. 11 (2002), 311-362, math.AG/0006026.

[25] Saito M.-H., Terajima H., Nodal curves and Riccati solutions of Painlevé equations, J. Math. Kyoto Univ. 44 (2004), 529-568, math.AG/0201225.

[26] Witte N.S., New transformations for Painlevé's third transcendent, Proc. Amer. Math. Soc. 132 (2004), 1649-1658, math.CA/0210019. 